\title{
Neck-related physical function, self-efficacy and coping strategies in patients with cervical radiculopathy: a randomized clinical trial of postoperative physiotherapy
}

J ohanna Wibault, Birgitta Öberg, Åsa Dedring, Håkan Löfgren, Peter Zsigmond, Liselott Persson, Margareta R. J onsson, Maria Andell and Anneli Peolsson

The self-archived version of this journal article is available at Linköping University Electronic Press:

http:// urn.kb.se/ resolve?urn=urn:nbn:se:liu:diva-122694

N.B.: When citing this work, cite the original publication.

Wibault, J., Öberg, B., Dedring, Å., Löfgren, H., Zsigmond, P., Persson, L., J onsson, M. R., Andell, M., Peolsson, A., (2017), Neck-related physical function, self-efficacy and coping strategies in patients with cervical radiculopathy: a randomized clinical trial of postoperative physiotherapy, J ournal of Manipulative and Physiological Therapeutics, 40(5), 330-339.

https:// dx.doi.org/ 10.1016/j.jmpt.2017.02.012

Original publication available at:

https://dx.doi.org/10.1016/j.jmpt.2017.02.012

Copyright: Elsevier

http:// www.elsevier.com/

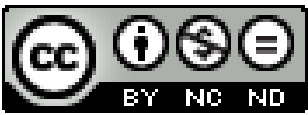




\section{Neck-related physical function, self-efficacy and coping strategies in patients with cervical radiculopathy - a randomized clinical trial of postoperative physiotherapy Abstract}

Objective: To compare postoperative rehabilitation with structured physiotherapy to standard approach in patients with cervical radiculopathy (CR) in a prospective randomized study at 6 months follow-up based on measures of neck-related physical function, self-efficacy and coping strategies.

Methods: Patients with persistent CR and scheduled for surgery $(n=202)$ were randomized to structured postoperative physiotherapy or standard postoperative approach. Structured postoperative physiotherapy combined neck-specific exercises with a behavioral approach. Baseline, 3-month, and 6-month evaluations included questionnaires and clinical examinations. Neck muscle endurance, active cervical range of motion, self-efficacy, pain catastrophizing (CSQ_CAT), perceived control over pain, and ability to decrease pain were analyzed for between-group differences using complete case and per-protocol approaches.

Results: No between-group difference was reported at the 6-month follow-up ( $p=0.05$ to 0.99), but all outcomes had improved from baseline $(\mathrm{p}<0.001)$. Patients undergoing structured postoperative physiotherapy with $\geq 50 \%$ attendance to treatment sessions showed larger improvements in CSQ_CAT $(\mathrm{p}=0.04)$ during the rehabilitation period from 3 to 6 months after surgery compared to the patients who received standard postoperative approach.

Conclusions: No between-group difference was found at 6 months after surgery based on measures of neck-related physical function, self-efficacy and coping strategies. However, the results confirm that neck-specific exercises are tolerated by patients with CR after surgery and may suggest a benefit from combining surgery with structured postoperative physiotherapy for patients with CR. 


\section{Introduction}

Cervical radiculopathy (CR) due to disc disease is characterized by radicular neck and arm pain, with associated neurological impairments. ${ }^{1,2}$ This condition often leads to disability, reduced health, and sickness-related absence from work. ${ }^{3}$

Patients who experience persistent symptoms may be referred for surgical treatment, ${ }^{4}$ which reportedly has overall good effects on arm pain and neurology. ${ }^{4,5}$ However, the effects of surgery on neck functioning are more uncertain, with studies showing persistent patientreported disability as measured with the Neck Disability Index (NDI), ${ }^{6,7}$ as well as impairments in neck muscle strength, neck muscle endurance (NME), and cervical active range of motion (cAROM) after surgery. ${ }^{6,8,9}$

Neck-specific exercises and cognitive behavioral treatments may be beneficial in other chronic neck pain disorders, ${ }^{10,11}$ and structured physiotherapy has been suggested as treatment before as well as after surgery to improve clinical outcomes in patients with CR. ${ }^{6-8 \text {, }}$ 12, 13 However, there is currently a lack of randomized clinical trials (RCTs) of postoperative physiotherapy in patients with CR to inform evidence-based clinical guidelines for the treatment of these patients. ${ }^{4}$

The present study aimed to compare rehabilitation with structured postoperative physiotherapy (SPT) versus standard postoperative approach (SA) in patients with CR based on measures of neck-related physical function, self-efficacy, and coping strategies at 6 months after surgery. 


\section{Methods}

\section{Design}

We designed a multi-center RCT of postoperative physiotherapy in patients with CR, and the study was performed February 2009 until December 2014. Patients were allocated in a one-toone ratio to parallel groups, and outcome measures were collected from independent blinded evaluators. Do to the nature of the study the treating physiotherapist and the patient was not blinded for treatment allocation. This study was approved by the regional ethical review board (Dnr M126-08) and performed in accordance with the Helsinki declaration. The protocol was registered with the Clinical Trial Identifier (NCT01547611), and has been previously published. ${ }^{14}$ Our previously reported results showed no between-group differences at the 6month follow-up with regard to the primary outcome NDI, neck and arm pain on the visual analogue scale (VAS), or global outcome (manuscript submitted). In this second report, we present the results for secondary outcome measures evaluating neck-related physical function, self-efficacy, and coping strategies. No serious harms or unintended effects was reported of the postoperative care.

\section{Participants}

We recruited patients who experienced persistent CR symptoms and were referred for surgery at four spinal centers in the south of Sweden between February 2009 and November 2012. Inclusion criteria were persistent CR symptoms for at least two months (median arm pain duration 12 months, 25 and 75 percentiles, 9 to 24 months), unsatisfactory improvements following non-surgical treatments, and magnetic resonance imaging (MRI) findings compatible with verified disc disease. Exclusion criteria were previous cervical surgery, cervical column fracture or traumatic subluxation, myelopathy, malignancy or spinal tumor, spinal infection, systematic disease implying a contraindication to an extensive rehabilitation, 
fibromyalgia or generalized myofascial pain, persistent or recurrent severe back pain, diagnosed mental disorder, drug or alcohol addiction and lack of fluency in Swedish. The study included a total of 202 patients who gave their informed consent. The mean age was 50 years, (SD 8.4 years), and 52\% were men. One patient was excluded because surgery was cancelled $(n=201)$ (Fig. 1). The patients were preoperatively randomized to receive either SPT or SA after surgery. The central project leader, who was not involved in any treatments or measurements simply randomized the patients using a random computer list developed by a statistician.

\section{Surgery and standard postoperative care at the spinal centers}

Patients were operated using anterior cervical decompression and fusion (ACDF) $(n=163)$ or posterior cervical foraminotomy (PCF) with or without laminectomy $(n=38)$. The disc(s) and osteophyte(s) were removed and the segments were fused using a standard cage(s) at each spinal center. The cage(s) was filled with bone substitute or autologous bone collected during decompression. Two-level $(n=62)$ or three-level fusions $(n=2)$ usually included the use of an anterior plate to achieve primary stability. PCF was performed without fusion at one-level ( $n=7)$, two levels $(n=15)$, or three or more levels $(n=16)$. During the first six weeks after surgery, all patients received the same standard postoperative care at the spinal centers, which included advice regarding good posture and ergonomics, recommendations to avoid certain activities and movements, and instructions for shoulder mobility exercises. After six weeks, patients returned to the spinal center for one routine visit to see the surgeon and the physiotherapist. On that occasion, patients were examined and given instructions to perform active exercises for cervical range of motion. 


\section{Interventions in the RCT}

\section{Structured postoperative physiotherapy (SPT)}

Following the six-week post-surgical visit to the spinal centers to see the surgeon and physiotherapist, patients randomized to SPT were referred to receive outpatient care from a physiotherapist. A nearby physiotherapist was selected from a total of 45 physiotherapists who were included in the study and instructed in the rehabilitation content by the project leader. SPT sessions combined exercise therapy for the neck, trunk and scapula muscles with a cognitive behavioral approach to pain and stress management (Fig. 2). ${ }^{14}$ Graded neckspecific exercises aimed to first activate the deep neck muscles and to then increase neuromuscular control and endurance. ${ }^{15}$ Swedish physiotherapists have basic knowledge of cognitive-behavioral treatments, and the included physiotherapists additionally completed a short practical training session with the project leader. Patients visited their physiotherapist once weekly during week 6 to 12, and twice weekly thereafter for a maximum of 20 weeks. Low-load endurance training for the neck, trunk and scapula was performed using a weighted pulley, an incline board, and resistive exercise bands, and these exercises were individually progressed. Patients were encouraged to perform home exercises between sessions and after discharge, as well as to increase their overall activity level (Fig. 2). The physiotherapists reported patient attendance to treatment sessions to the project leader by telephone or mail.

\section{Standard postoperative approach (SA)}

SA was provided in accordance with Swedish usual postoperative care. After the routine visit to the surgeon and physiotherapist at the spinal centers at six weeks after surgery, patients were advised to contact their primary healthcare center for additional treatments when needed (Fig. 2). The postoperative treatments were pragmatic and may have included postoperative physiotherapy. 


\section{Data collection}

Before surgery and at 3- and 6-month post-surgical follow-ups, the patients were assessed using a set of questionnaires and clinical examinations. The questionnaires were sent by postal mail, and were collected by an independent investigator blinded to the randomization. Patients who were late in returning the questionnaires were sent postal reminders. Clinical examinations included assessment of neck-related physical function and hand strength, static and dynamical balance tests, and a neurological examination was performed at baseline. These exams were performed by four independent physiotherapists at each spinal center who were blinded to patient randomization. ${ }^{14}$ Surgical data were collected from medical journals.

\section{Background variables}

The questionnaire completed collected the following background variables age, sex, duration of symptoms in the neck and arm (in months), smoking (yes/no), sickness-related absence due to neck and arm symptoms (yes/no), and preoperative use of physiotherapy treatments (yes/no). Baseline questionnaires also obtained preoperative scores on the NDI, and VAS neck and arm pain ${ }^{16}$ (Table 1). Surgical data included the type of surgery (ACDF versus PCF) and the number of operated levels (Table 1).

\section{Outcomes measures}

\section{Clinical measures of neck-related physical function}

With the patient in an upright seated position, cAROM in the sagittal, transverse and frontal planes of movement was measured in degrees. We used a cervical range of motion (CROM) device (Performance Attainment Associated, Roseville, MN), ${ }^{17}$ with reportedly good reliability in patients with neck pain. ${ }^{18} \mathrm{NME}$ during flexion and extension was evaluated in seconds. Patients were in the supine position for measuring NME of the cervical anterior 
muscles, and in prone position for measuring NME of the cervical posterior muscles with a 2kg weight for women and a 4-kg weight for men. ${ }^{19}$ Such NME measurements have shown good to acceptable reliability in patients with non-specific neck pain. ${ }^{20,21}$

\section{Self-efficacy and coping strategies}

The Swedish version of the Self-Efficacy Scale (SES) was used to evaluate each patient's confidence in their own ability to perform 20 daily activities despite their pain. Responses ranged from “not at all confident” (0) to "very confident” (10) and response values were summed to a total score of 0 -200), ${ }^{22,23}$ with higher scores indicating higher self-efficacy. Each patient's current use of coping strategies was assessed using three subscales of the Swedish version of the Coping Strategy Questionnaire. ${ }^{24}$ The catastrophizing subscale (CSQ_CAT) was used to evaluate current use of negative thinking as a reaction to pain (score 0-36 with higher score indicating higher level of pain catastrophizing). The CSQ_COP subscale was used to evaluate the self-perceived effectiveness of coping strategies to control pain, and the CSQ_ADP subscale assessed the ability to decrease pain. Both the CSQ_COP and CSQ_ADP are single-item scales scored from 0-6 with higher scores indicating greater ability to decrease pain using coping strategies and more pain control respectively.

\section{Statistical analysis}

Sample size estimation was based on a between-group difference of $10 \%$ in the primary outcome NDI and 202 patients were recruited. ${ }^{14}$ Descriptive statistics were reported as mean and standard deviation (SD), median and interquartile range (IQR), or proportions. If one item was missing from the SES, it was substituted with the average item score of the instrument for that patient. If multiple items were missing, the patient was excluded from analysis. Betweengroup differences in background variables (Table 1), as well as in outcome measures at baseline (Table 2) were analyzed using parametric and non-parametric statistics depending on 
the data level. For outcome measures, we first performed a complete case analysis. Next, patients in the SPT group who had $\geq 50 \%$ attendance to treatment sessions (SPT $\geq 50 \%$ ) were compared with patients who received SA in a per-protocol approach. Between-group differences in outcomes, and in changes in outcomes form before surgery to 6 months were investigated using repeated measures analysis of variance (ANOVA) with GreenhouseGeisser correction depending on sphericity. Non-normally distributed interval data and ordinal data were previously log-transformed to fulfill ANOVA assumptions. Next, betweengroup differences in outcome score changes from baseline to 6 months and during the rehabilitation period from 3 to 6 months after surgery were studied in a simple analysis using the independent t-test for interval data and the Mann-Whitney-U test for ordinal data. Statistical analyses were performed using the statistical software package SPSS version 20.0 (IBM Corporation, New York, USA). A $p$ value of $<0.05$ was considered to indicate statistical significance.

\section{Results}

\section{Differences between the treatment groups at follow-up}

Complete case analysis revealed no difference in outcome measures between the treatment groups, with $p$ values ranging from 0.12 to 0.99 (Table 3). From baseline to the 6-month follow-up, all outcome measures improved significantly $(\mathrm{p}<0.001)$, except from ROM in the transversal plan $(\mathrm{p}=0.35$ ) (Table 3). Similar results were obtained using a per-protocol approach (Table 3). Simple analysis of outcome score changes from baseline to 6 months and during the rehabilitation period from 3 months to 6 months after surgery, showed no difference between the treatment groups in a complete case analysis, with $p$ values ranging from 0.10 to 0.97 . A per-protocol approach revealed significantly larger improvements in CSQ_CAT during the rehabilitation period (from 3 to 6 months after surgery) in SPT $\geq 50 \%$ 
compared to patients who received SA $(p=0.04)$. Table 2 presents descriptive statistics for the outcome measures in the intervention groups at baseline, and at the 3- and 6-month follow-up.

\section{Treatment groups at baseline}

The treatment groups did not differ for background variables or surgical data in a complete case analysis ( $p$ values ranging from 0.08 to 0.99 ), with the exception that the SPT group showed a longer duration of neck and arm pain symptoms ( $p$ values ranging from 0.02 to 0.03). No difference was found in a per-protocol approach ( $p$ values ranging from 0.09 to 0.82). No difference was reported in outcome measures at baseline in a complete case analysis or a per-protocol approach (p ranging from 0.18 to 0.98 ).

\section{Patients lost to follow-up}

Patients lost to follow-up were excluded from the analysis due to missing data. The proportion of patients lost to follow-up for the different outcome measures did not differ between the treatment groups ( $p=0.34$ to 0.61 ) (Fig. 1). Compared with the patients included in the analysis, the patients lost to follow-up had significantly lower NME in flexion and extension, and lower ROM in the frontal plan of movement at baseline ( $p=0.01$ to 0.03 ). Patients lost to follow-up did not show significantly different measures of self-efficacy and coping strategies before surgery ( $p=0.08$ to 0.45 ).

\section{Attendance to treatment sessions in patients who received SPT}

The physiotherapists involved in this study reported that $67 \%$ of the patients showed a $\geq 50 \%$ attendance rate to treatment sessions (SPT $\geq 50 \%, n=67$, mean age 51 years, SD 8.2 years; 33 men and 34 women). The remaining 34 patients were excluded in the per-protocol analysis 
approach (mean age 48 years, SD 8.1 years; 18 men and 16 women). The reasons given included lack of time for rehabilitation and difficulties missing work $(n=19)$, not starting the rehabilitation intervention for unknown reasons $(\mathrm{n}=10)$. Reports on attendance rate were missing for 5 patients. Compared to patients in the SPT $\geq 50 \%$ group $(n=67)$, the excluded patients ( $\mathrm{n}=34)$ showed a higher baseline rate of smokers ( $41 \%$ versus $16 \%, p=0.005)$, and lower baseline SES scores $(p=0.04)$. No other difference was found at baseline $(p=0.11$ to $0.92)$.

\section{Discussion}

The present study was the first RCT of postoperative physiotherapy in patients with CR due to cervical disc disease and is thereby unique. Thus the present study is of great clinical importance resulting in indications how to improve future postoperative care. At the 6-month follow-up, repeated measures ANOVA revealed no between-group differences in measures of neck-related physical function, self-efficacy and coping strategies. However, with simple analysis of outcome score changes during the rehabilitation period from 3 to 6 months after surgery, we observed significantly larger improvements in pain catastrophizing in SPT $\geq 50 \%$ compared to patients who received SA. The 6-month results in the present study should be interpreted cautiously since inconsistent findings were reported due to small subgroups of patients.

From baseline to 6 months after surgery, both treatment groups showed significant improvements in clinical measures of neck-related physical function. NME is known to differ between men and women ${ }^{19}$ and were reported separately. There was a tendency for NME in extension to improve more in men and women who received SPT/ SPT $\geq 50 \%$ compared to patients who received SA but no significant between-group difference was found. Moreover, 
interpretation of these results is limited by our restricted knowledge of systematic and random errors for measures of NME and cAROM in patients with $\mathrm{CR},{ }^{25}$ as well as regarding the amplitude of changes that are of clinical importance. Previous studies have shown improvements in NME in patients with CR after surgery, ${ }^{6,13,26}$ as well as after rehabilitation with physiotherapy alone. ${ }^{13}$ These results suggest that different interventions can improve NME in patients with CR. On the other hand, conflicting results have been reported regarding cAROM improvement after surgery for CR. ${ }^{6,13,27}$ Studies have more frequently emphasized post-surgical impairments in measures of NME and cAROM ${ }^{6,8}$ compared with reference values in neck-healthy individuals. ${ }^{19,28}$

From baseline to the 6-month follow-up, both treatment groups reported significant improvements in self-efficacy and coping strategies. The improvements in pain catastrophizing in SPT $\geq 50 \%$ were comparable to those reported by lumbar fusion patients undergoing postoperative psychomotor therapy, ${ }^{29}$ suggesting that similar effect mechanisms may be at play, although the intervention contents differed. ${ }^{29}$ Reduced pain catastrophizing was reportedly suggested to mediate the effects of both exercise therapy and cognitive behavioral treatments on function and pain among patients with chronic low back pain. ${ }^{30}$ The importance of regular physiotherapist supervision for increased treatment effects following exercise therapy has been suggested. ${ }^{31}$

The presently described RCT of postoperative physiotherapy in patients with CR included physiotherapists with a broad spectrum of levels of experience, competence, and specialized knowledge mirroring the clinical set-up of most departments. Future studies should investigate whether the involvement of physiotherapists who are specialized in the management of musculoskeletal disorders can further improve measures of neck-related physical function, self-efficacy and coping strategies in patients with CR after surgery. Future 
studies should also identify patients who might particularly benefit from extended postoperative physiotherapy e.g patients with a poor surgical outcome and investigate the benefit of SPT compared to SA. The results from our current study will guide such future research.

More generally, our broad patient assessment including measures of neck-related physical function and psychosocial factors improved the description of treatment outcomes, as was previously suggested by Wibault et al. ${ }^{32}$ The results show that neck-specific exercises are tolerated by patients with CR after surgery and they may suggest a benefit from structured postoperative physiotherapy. Future studies should investigate the long-term effects of SPT in patients with CR considering that some effects may be delayed up to one year after surgery. ${ }^{12}$ Engquist et al. ${ }^{12}$ and Peolsson et al. ${ }^{13}$ performed a study investigating the additional effect of surgery to structured physiotherapy in CR patients due to disc disease and reported a faster neck pain reduction, but otherwise no differences in either self-reported or physical measures at the two year follow-up. They ${ }^{12,13}$ concluded that physiotherapy should precede a decision of surgery. The present study is the first RCT of postoperative physiotherapy in patients with $\mathrm{CR}$, and the results indicate the benefit of structured postoperative physiotherapy, supporting the importance of structured treatment in patients with CR earlier concluded. ${ }^{12,13}$

\section{Limitations}

The sample size estimation in the present study was based on the primary outcome NDI. A type II error cannot be excluded when analyzing between-group differences in secondary outcome measures. $^{33}$ 
For the different outcome measures, the proportions of patients lost to follow-up varied between $6-18 \%$ at 3 months, and between $15-19 \%$ at 6 months. However, these proportions did not significantly differed between the treatment groups. Moreover, baseline measures of self-efficacy and coping strategies did not significantly differ between patients who responded and did not responded to the questionnaires at follow-up. Thus, the patients lost to follow-up were not considered to have compromised the generalizability of the results.

At the 3-month follow-up, we did not perform NME measurement due to restricted loading of the neck following fusion surgery, and we did not evaluate SES to limit the number of questions in the questionnaire. Thus, another limitation of the study was the lack of evaluation of within-group differences in SES and NME during the rehabilitation period from 3 to 6 months after surgery.

\section{Conclusions}

In conclusion, here we describe the first prospective RCT comparing postoperative rehabilitation with SPT or SA in patients with CR. Our results showed no difference between the treatment groups at the 6-month follow-up based on measures of neck-related physical function, self-efficacy, and coping strategies. During the rehabilitation period (from 3 to 6 months after surgery), we did find a larger reduction in pain catastrophizing in SPT $\geq 50 \%$ compared to patients who received SA. The results confirm that neck-specific exercises are tolerated by patients with CR after surgery; and they may suggest a benefit from combining surgery with structured postoperative physiotherapy in patients with CR. Future studies should aim to identify patients with CR who might benefit more from extended postoperative physiotherapy, as well as investigate the possibility of developing rehabilitation protocols that can further improve neck-related physical function, self-efficacy, and coping strategies in patients with CR. 


\section{Funding and conflict of interest}

The authors acknowledge financial support from the Swedish Research Council, the Swedish Society of Medicine, the Medical Research Council of Southeast Sweden, Region

Östergötland, Lions, and Futurum (Academy of Health and Care, Region Jönköping County).

The authors have no conflicts of interest to report.

\section{References}

1. Carette S, Fehlings MG. Clinical practice. Cervical radiculopathy. $N$ Engl J Med 2005;353:392-9.

2. Caridi JM, Pumberger M, Hughes AP. Cervical radiculopathy: a review. HSS journal : the musculoskeletal journal of Hospital for Special Surgery 2011;7:265-72.

3. Daffner SD, Hilibrand AS, Hanscom BS, Brislin BT, Vaccaro AR, Albert TJ. Impact of neck and arm pain on overall health status. Spine 2003;28:2030-5.

4. Bono CM, Ghiselli G, Gilbert TJ, Kreiner DS, Reitman C, Summers JT, et al. An evidence-based clinical guideline for the diagnosis and treatment of cervical radiculopathy from degenerative disorders. Spine J 2011;11:64-72.

5. Löfgren H, Johansen F, Skogar O, Levander B. Reduced pain after surgery for cervical disc protrusion/stenosis: a 2 year clinical follow-up. Disabil Rehabil 2003;25:1033-43.

6. Peolsson A, Vavruch L, Oberg B. Disability after anterior decompression and fusion for cervical disc disease. Advances in Physiotherapy 2002;4:111-24.

7. Hermansen A, Hedlund R, Vavruch L, Peolsson A. A comparison between the carbon fiber cage and the cloward procedure in cervical spine surgery: a ten- to thirteen-year followup of a prospective randomized study. Spine 2011;36:919-25.

8. Hermansen AM, Cleland JA, Kammerlind AS, Peolsson AL. Evaluation of physical function in individuals 11 to 14 years after anterior cervical decompression and fusion surgery-a comparison between patients and healthy reference samples and between 2 surgical techniques. J Manipulative Physiol Ther 2014;37:87-96.

9. Ylinen JJ, Savolainen S, Airaksinen O, Kautiainen H, Salo P, Hakkinen A. Decreased strength and mobility in patients after anterior cervical diskectomy compared with healthy subjects. Arch Phys Med Rehabil 2003;84:1043-7.

10. Gross A, Kay TM, Paquin JP, Blanchette S, Lalonde P, Christie T, et al. Exercises for mechanical neck disorders. Cochrane Database Syst Rev 2015;1:CD004250.

11. Monticone M, Ambrosini E, Cedraschi C, Rocca B, Fiorentini R, Restelli M, et al. Cognitive-behavioral Treatment for Subacute and Chronic Neck Pain: A Cochrane Review. Spine 2015;40:1495-504. 
12. Engquist M, Löfgren H, Öberg B, Holtz A, Peolsson A, Soderlund A, et al. Surgery versus nonsurgical treatment of cervical radiculopathy: a prospective, randomized study comparing surgery plus physiotherapy with physiotherapy alone with a 2-year follow-up. Spine 2013;38:1715-22.

13. Peolsson A, Soderlund A, Engquist M, Lind B, Löfgren H, Vavruch L, et al. Physical function outcome in cervical radiculopathy patients after physiotherapy alone compared with anterior surgery followed by physiotherapy: a prospective randomized study with a 2-year follow-up. Spine 2013;38:300-7.

14. Peolsson A, Öberg B, Wibault J, Dedering A, Zsigmond P, Bernfort L, et al. Outcome of physiotherapy after surgery for cervical disc disease: a prospective randomised multi-centre trial. BMC Musculoskelet Disord 2014;15:34.

15. Landen Ludvigsson M, Peterson G., Peolsson A.; Available from: www.divaportal.org/smash/get/diva2:785214/FULLTEXT02.pdf.

16. Peolsson A, Oberg B, Wibault J, Dedering A, Zsigmond P, Bernfort L, et al. Outcome of physiotherapy after surgery for cervical disc disease: a prospective randomised multi-centre trial. BMC Musculoskelet Disord 2014;15:34.

17. Youdas JW, Carey JR, Garrett TR. Reliability of measurements of cervical spine range of motion--comparison of three methods. Phys Ther 1991;71:98-104.

18. de Koning CH, van den Heuvel SP, Staal JB, Smits-Engelsman BC, Hendriks EJ. Clinimetric evaluation of active range of motion measures in patients with non-specific neck pain: a systematic review. Eur Spine J 2008;17:905-21.

19. Peolsson A, Almkvist C, Dahlberg C, Lindqvist S, Pettersson S. Age- and sex-specific reference values of a test of neck muscle endurance. $J$ Manipulative Physiol Ther 2007;30:171-7.

20. Peolsson A. HC, Albinsson A-K., Engdahl S., Kvist J. . Test position and reliability in measurements of dorsal neck muscle endurance. Adv Physiotherapy 2007;9:181-9.

21. de Koning CH, van den Heuvel SP, Staal JB, Smits-Engelsman BC, Hendriks EJ. Clinimetric evaluation of methods to measure muscle functioning in patients with nonspecific neck pain: a systematic review. BMC Musculoskelet Disord 2008;9:142.

22. Altmaier EM RD, Kao CF, Lehmann TR, Weinstein JN, . Role of self-efficacy in rehabilitation outcome among chronic low back pain patients. Journal of Counseling Psychology 1993;40:335-9.

23. Bunketorp L, Carlsson J, Kowalski J, Stener-Victorin E. Evaluating the reliability of multi-item scales: a non-parametric approach to the ordered categorical structure of data collected with the Swedish version of the Tampa Scale for Kinesiophobia and the SelfEfficacy Scale. J Rehabil Med 2005;37:330-4.

24. Jensen IL, SJ. Coping strategies questionnaire: reliability of the Swedish version of the CSQ. Scandinavian Journal of Behavioral Therapy 1993;22:139-45. 
25. Gatchel RJ, Mayer TG. Testing minimal clinically important difference: consensus or conundrum? Spine J 2010;10:321-7.

26. Peolsson A, Kjellman G. Neck muscle endurance in nonspecific patients with neck pain and in patients after anterior cervical decompression and fusion. $J$ Manipulative Physiol Ther 2007;30:343-50.

27. Hilibrand AS, Balasubramanian K, Eichenbaum M, Thinnes JH, Daffner S, Berta S, et al. The effect of anterior cervical fusion on neck motion. Spine (Phila Pa 1976) 2006;31:1688-92.

28. Youdas JW, Garrett TR, Suman VJ, Bogard CL, Hallman HO, Carey JR. Normal range of motion of the cervical spine: an initial goniometric study. Phys Ther 1992;72:770-80. 29. Abbott AD, Tyni-Lenne R, Hedlund R. Early rehabilitation targeting cognition, behavior, and motor function after lumbar fusion: a randomized controlled trial. Spine 2010;35:848-57.

30. Smeets RJ, Vlaeyen JW, Kester AD, Knottnerus JA. Reduction of pain catastrophizing mediates the outcome of both physical and cognitive-behavioral treatment in chronic low back pain. J Pain 2006;7:261-71.

31. Hayden JA, van Tulder MW, Tomlinson G. Systematic review: strategies for using exercise therapy to improve outcomes in chronic low back pain. Ann Intern Med 2005;142:776-85.

32. Wibault J, Öberg B, Dedering A, Löfgren H, Zsigmond P, Persson L, et al. Individual factors associated with neck disability in patients with cervical radiculopathy scheduled for surgery: a study on physical impairments, psychosocial factors, and life style habits. Eur Spine J 2014;23:599-605.

33. Bailey CS, Fisher CG, Dvorak MF. Type II error in the spine surgical literature. Spine 2004;29:1146-9.

\section{Figure legends}

Fig 1: Patient flow diagram for the study inclusion. A complete case analysis and a perprotocol approach (comparing patients with at least $50 \%$ attendance to treatment sessions in the structured postoperative physiotherapy group to patients who received standard postoperative approach) were performed.

Fig 2: Content of structured postoperative physiotherapy (SPT), and standard postoperative approach (SA). 
Table 1: Background variables and surgical data for the study participants

Background variables

Age in years, mean (SD)

Male sex, \% (n)

Duration of neck pain in months, median (IQR)

Smoking, \% (n)

Sickness-related absence due to neck/ arm symptoms, \% (n) 178

Preoperative use of physiotherapy treatments, \% (n)

NDI, median (IQR)

Neck pain VAS, median (IQR)

Arm pain VAS, median (IQR)
Duration of arm pain in months, median (IQR)

n Scores

$201 \quad 50.0(8.4)$

$201 \quad 52(105)$

$173 \quad 14.0(27.0)$

$166 \quad 12.0(15.3)$

$194 \quad 24(49)$

$56(100)$

66 (124)

$192 \quad 42.0(20.0)$

$196 \quad 60.5(36.0)$

$193 \quad 54.0(47.0)$

\section{Surgical data}

ACDF, \% (n)

201

$81(163)$

201

$53(106)$

38 (77)

$9(18)$

2-levels surgery

$\geq 3$-levels surgery

SD: standard deviation; IQR: interquartile range; NDI: Neck Disability Index (0-100\%); VAS: visual analogue scale (0-100 mm); ACDF: anterior cervical decompression and fusion. 
Table 2: Neck-related physical function, self-efficacy, and coping strategies at baseline, and at the 3- and 6-month follow-ups in the intervention groups structured postoperative physiotherapy (SPT) and standard postoperative approach (SA). Data is also presented for patients with at least $50 \%$ attendance to treatment sessions (SPT $\geq 50 \%$ ).

\section{Descriptive statistics}

\begin{tabular}{|c|c|c|c|c|c|c|c|c|c|}
\hline & \multicolumn{3}{|c|}{ Baseline } & \multicolumn{3}{|c|}{3 months } & \multicolumn{3}{|c|}{6 months } \\
\hline & $\begin{array}{c}\text { Structured } \\
\text { postoperative } \\
\text { physiotherapy } \\
(\mathbf{n}=101) \\
\end{array}$ & $\begin{array}{c}\geq \mathbf{5 0 \%} \\
\text { attendance } \\
(\mathrm{n}=67)\end{array}$ & $\begin{array}{l}\text { Standard } \\
\text { approach } \\
(\mathbf{n}=100)\end{array}$ & ${ }^{1} \mathrm{SPT}$ & $\begin{array}{c}{ }^{2} \text { SPT } \geq \\
50 \%\end{array}$ & ${ }^{3} \mathrm{SA}$ & ${ }^{1} \mathrm{SPT}$ & $\begin{array}{c}{ }^{2} \text { SPT } \geq \\
50 \%\end{array}$ & ${ }^{3} \mathrm{SA}$ \\
\hline \multicolumn{10}{|c|}{$\begin{array}{l}\text { NECK-RELATED } \\
\text { PHYSICAL } \\
\text { FUNCTION }\end{array}$} \\
\hline $\begin{array}{l}\text { Total, n } \\
\text { NME flexion, } \\
\text { median (IQR) }\end{array}$ & $\begin{array}{c}98 \\
25.0 \\
(48)\end{array}$ & $\begin{array}{c}64 \\
27.5 \\
(62)\end{array}$ & $\begin{array}{c}94 \\
31.0 \\
(41)\end{array}$ & & & & $\begin{array}{c}84 \\
48.5 \\
(70)\end{array}$ & $\begin{array}{c}59 \\
57.0 \\
(85)\end{array}$ & $\begin{array}{c}81 \\
56.0 \\
(54)\end{array}$ \\
\hline Men, n & $\begin{array}{c}49 \\
\mathbf{4 5 . 0} \\
(96)\end{array}$ & $\begin{array}{c}31 \\
\mathbf{5 7 . 0} \\
(93)\end{array}$ & $\begin{array}{c}52 \\
44.0 \\
(55)\end{array}$ & & & & $\begin{array}{c}40 \\
\mathbf{8 5 . 0} \\
(109)\end{array}$ & $\begin{array}{c}27 \\
\mathbf{9 4 . 0} \\
(120)\end{array}$ & $\begin{array}{c}43 \\
\mathbf{6 4 . 0} \\
(88)\end{array}$ \\
\hline Women, n & $\begin{array}{c}49 \\
21.0 \\
(28)\end{array}$ & $\begin{array}{c}33 \\
19.0 \\
(29)\end{array}$ & $\begin{array}{c}43 \\
\mathbf{2 2 . 0} \\
(31)\end{array}$ & & & & $\begin{array}{c}39 \\
32.0 \\
(36)\end{array}$ & $\begin{array}{c}27 \\
36.0 \\
(40)\end{array}$ & $\begin{array}{c}36 \\
36.0 \\
(48)\end{array}$ \\
\hline $\begin{array}{l}\text { Total, n } \\
\text { NME extension, } \\
\text { median (IQR) }\end{array}$ & $\begin{array}{c}98 \\
32.0 \\
(106)\end{array}$ & $\begin{array}{c}64 \\
32.5 \\
(156)\end{array}$ & $\begin{array}{c}94 \\
52.5 \\
(84)\end{array}$ & & & & $\begin{array}{c}84 \\
95.5 \\
(147)\end{array}$ & $\begin{array}{c}59 \\
\mathbf{9 4 . 0} \\
(143)\end{array}$ & $\begin{array}{c}81 \\
\mathbf{8 0 . 0} \\
(139)\end{array}$ \\
\hline Men, n & $\begin{array}{c}49 \\
43.0 \\
(189)\end{array}$ & $\begin{array}{c}31 \\
\mathbf{8 9 . 0} \\
(248)\end{array}$ & $\begin{array}{c}51 \\
\mathbf{8 9 . 0} \\
(102)\end{array}$ & & & & $\begin{array}{c}40 \\
\mathbf{1 2 4 . 0} \\
(184)\end{array}$ & $\begin{array}{c}27 \\
\mathbf{1 6 5 . 0} \\
(184)\end{array}$ & $\begin{array}{c}36 \\
\mathbf{8 4 . 0} \\
(176)\end{array}$ \\
\hline Women, n & $\begin{array}{c}49 \\
24.0 \\
(52) \\
\end{array}$ & $\begin{array}{c}33 \\
22.0 \\
(52)\end{array}$ & $\begin{array}{c}43 \\
35.0 \\
(57) \\
\end{array}$ & & & & $\begin{array}{c}39 \\
\mathbf{8 2 . 0} \\
(76)\end{array}$ & $\begin{array}{c}27 \\
\mathbf{8 2 . 0} \\
(63)\end{array}$ & $\begin{array}{c}36 \\
\mathbf{6 6 . 5} \\
(120) \\
\end{array}$ \\
\hline
\end{tabular}




\begin{tabular}{|c|c|c|c|c|c|c|c|c|c|}
\hline $\begin{array}{l}\text { Total, } \mathrm{n} \\
\text { cAROM sagittal, } \\
\text { mean (SD) }\end{array}$ & $\begin{array}{c}100 \\
83(27.7)\end{array}$ & $85(28.2)$ & 83 (25.7) & $\begin{array}{c}95 \\
85(23.9)\end{array}$ & $\begin{array}{c}65 \\
88(21.2)\end{array}$ & 86 (19.0) & 93 (21.7) & $94(20.6)$ & $\begin{array}{c}82 \\
92(21.6)\end{array}$ \\
\hline $\begin{array}{l}\text { cAROM transverse, } \\
\text { mean (SD) }\end{array}$ & 54 (19.5) & 54 (18.9) & 55 (15.9) & 53 (18.5) & 53 (16.6) & 55 (16.3) & 57 (16.0) & $57(16.4)$ & 56 (16.2) \\
\hline $\begin{array}{l}\text { cAROM frontal } \\
\text { mean }(S D)\end{array}$ & $100(26.4)$ & $102(27.0)$ & $100(24.7)$ & $101(25.7)$ & 104(22.4 & 107(19.5) & 108 (23.9) & $111(24.9)$ & $111(20.3)$ \\
\hline \multicolumn{10}{|c|}{$\begin{array}{l}\text { SELF-EFFICACY AND } \\
\text { COPING } \\
\text { STRATEGIES }\end{array}$} \\
\hline $\begin{array}{l}\text { Total, n } \\
\text { SES, median (IQR) }\end{array}$ & $\begin{array}{c}97 \\
\mathbf{1 2 9 . 0}(62)\end{array}$ & $\begin{array}{c}64 \\
137.5(66)\end{array}$ & $\begin{array}{c}96 \\
\mathbf{1 2 9 . 5}(55)\end{array}$ & & & & $\begin{array}{c}90 \\
\mathbf{1 7 1 . 0}(57)\end{array}$ & $\begin{array}{c}65 \\
\mathbf{1 7 7 . 0}(42)\end{array}$ & $\begin{array}{c}81 \\
177.0(61)\end{array}$ \\
\hline $\begin{array}{l}\text { Total, n } \\
\text { CSQ_CAT, median } \\
\text { (IQR) }\end{array}$ & $\begin{array}{c}96 \\
\mathbf{1 3 . 0}(12)\end{array}$ & $\begin{array}{c}63 \\
\mathbf{1 2 . 0}(13)\end{array}$ & $\begin{array}{c}96 \\
\mathbf{1 3 . 0}(11)\end{array}$ & $\begin{array}{c}87 \\
7.0(10)\end{array}$ & $\begin{array}{c}64 \\
7.0(9)\end{array}$ & $\begin{array}{c}78 \\
6.5(14)\end{array}$ & $\begin{array}{c}85 \\
5.0(9)\end{array}$ & $\begin{array}{c}63 \\
\mathbf{4 . 0}(7)\end{array}$ & $\begin{array}{c}78 \\
\mathbf{6 . 5}(13)\end{array}$ \\
\hline $\begin{array}{l}\text { Total, } \mathrm{n} \\
\text { CSQ_COP, median } \\
\text { (IQR) }\end{array}$ & $\begin{array}{c}98 \\
\mathbf{4 . 0}(2)\end{array}$ & $\begin{array}{c}64 \\
3.5(2)\end{array}$ & $\begin{array}{c}96 \\
\mathbf{3 . 0}(1)\end{array}$ & $\begin{array}{c}86 \\
\mathbf{4 . 0}(2)\end{array}$ & $\begin{array}{c}64 \\
\mathbf{4 . 0}(2)\end{array}$ & $\begin{array}{c}80 \\
\mathbf{4 . 0}(2)\end{array}$ & $\begin{array}{c}88 \\
\mathbf{4 . 0}(2)\end{array}$ & $\begin{array}{c}65 \\
\mathbf{5 . 0}(3)\end{array}$ & $\begin{array}{c}79 \\
\mathbf{5 . 0}(2)\end{array}$ \\
\hline $\begin{array}{l}\text { Total, } \mathrm{n} \\
\text { CSQ_ADP, median } \\
\text { (IQR) }\end{array}$ & $\begin{array}{c}97 \\
\mathbf{3 . 0}(2)\end{array}$ & $\begin{array}{c}63 \\
\mathbf{3 . 0}(2)\end{array}$ & $\begin{array}{c}96 \\
\mathbf{3 . 0}(1)\end{array}$ & $\begin{array}{c}85 \\
3.0(2)\end{array}$ & $\begin{array}{c}63 \\
\mathbf{4 . 0}(2)\end{array}$ & $\begin{array}{c}80 \\
\mathbf{4 . 0}(2)\end{array}$ & $\begin{array}{c}88 \\
3.5(2)\end{array}$ & $\begin{array}{c}65 \\
\mathbf{4 . 0}(2)\end{array}$ & $\begin{array}{c}79 \\
\mathbf{3 . 0}(2)\end{array}$ \\
\hline
\end{tabular}

${ }^{1}$ SPT: structured postoperative physiotherapy group; ${ }^{2}$ SPT $\geq 50 \%$ : patients with at least $50 \%$ attendance tot treatment sessions in the structured postoperative physiotherapy group; ${ }^{3} \mathrm{SA}$ : standard postoperative approach group

NME: neck muscle endurance as flexion and extension in seconds; cAROM: cervical range of motion in degrees; SES: self-efficacy; CSQ: coping strategy questionnaire; CSQ_CAT: catastrophizing subscale of CSQ; CSQ_COP: control over pain subscale; CSQ_ADP: ability to decrease pain subscale 
Table 3: Between-group differences in outcomes and changes from baseline to 6 months after surgery for measures of neck-related physical function, self-efficacy, and coping strategies in a complete case analysis and a per-protocol approach

\section{Complete case analysis}

\begin{tabular}{|c|c|c|c|c|c|c|c|c|}
\hline & $\begin{array}{c}\mathbf{N} \\
\mathrm{SPT}^{1} ; \\
\mathrm{SA}^{3}\end{array}$ & $\begin{array}{l}\text { Between- } \\
\text { group } \\
\text { differences }\end{array}$ & $\begin{array}{l}\text { Changes } \\
\text { over time }\end{array}$ & $\begin{array}{l}\text { Between-group } \\
\text { differences in } \\
\text { changes over } \\
\text { time }\end{array}$ & $\begin{array}{c}\mathbf{N} \\
\mathrm{SPT} \geq 50 \%{ }^{2} \\
\mathrm{SA}^{3}\end{array}$ & $\begin{array}{l}\text { Between- } \\
\text { group } \\
\text { differences }\end{array}$ & $\begin{array}{l}\text { Changes } \\
\text { over time }\end{array}$ & $\begin{array}{l}\text { Between-group } \\
\text { differences in } \\
\text { changes over } \\
\text { time }\end{array}$ \\
\hline Log (NME flexion) & $83 ; 77$ & $\begin{array}{c}\mathrm{F}(1,158) \\
0.11 \\
\mathbf{p}=\mathbf{0 . 7 5}\end{array}$ & $\begin{array}{c}\mathrm{F}(1,158) \\
36.50 \\
\boldsymbol{p}<\boldsymbol{0 . 0 0 1}\end{array}$ & $\begin{array}{c}\mathrm{F}(1,158) \\
0.59 \\
\mathbf{p}=\mathbf{0 . 4 4}\end{array}$ & $58 ; 77$ & $\begin{array}{c}\mathrm{F}(1,133) \\
0.16 \\
\mathbf{p}=\mathbf{0 . 9 0}\end{array}$ & $\begin{array}{c}\mathrm{F}(1,133) \\
36.44 \\
\boldsymbol{p}<\boldsymbol{0 . 0 0 1}\end{array}$ & $\begin{array}{c}\mathrm{F}(1,133) \\
1.32 \\
\mathbf{p}=\mathbf{0 . 2 5}\end{array}$ \\
\hline $\begin{array}{l}\text { Log (NME } \\
\text { extension) }\end{array}$ & $83 ; 76$ & $\begin{array}{c}\mathrm{F}(1,157) \\
0.001 \\
\mathbf{p}=\mathbf{0 . 9 9}\end{array}$ & $\begin{array}{c}\mathrm{F}(1,157) \\
25.94 \\
\boldsymbol{p}<\mathbf{0 . 0 0 1}\end{array}$ & $\begin{array}{c}\mathrm{F}(1,157) \\
0.78 \\
\mathbf{p}=\mathbf{0 . 3 8}\end{array}$ & $58 ; 76$ & $\begin{array}{c}\mathrm{F}(1,132) \\
0.07 \\
\mathbf{p}=\mathbf{0 . 7 9}\end{array}$ & $\begin{array}{c}\mathrm{F}(1,132) \\
29.64 \\
\boldsymbol{p}<\mathbf{0 . 0 0 1}\end{array}$ & $\begin{array}{c}\mathrm{F}(1,132) \\
1.42 \\
\mathbf{p}=\mathbf{0 . 2 4}\end{array}$ \\
\hline cAROM sagittal & $84 ; 79$ & $\begin{array}{c}F(1,161) \\
0.03 \\
\mathbf{p}=\mathbf{0 . 8 7}\end{array}$ & $\begin{array}{c}\mathrm{F}(1.6,259) \\
13.47 \\
\boldsymbol{p}<\mathbf{0 . 0 0 1}\end{array}$ & $\begin{array}{c}\mathrm{F}(1.6,259) \\
0.08 \\
\mathbf{p}=\mathbf{0 . 8 9}\end{array}$ & $60 ; 79$ & $\begin{array}{c}\mathrm{F}(1,137) \\
0.01 \\
\mathbf{p}=\mathbf{0 . 9 2}\end{array}$ & $\begin{array}{c}\mathrm{F}(1.5,212) \\
10.99 \\
\boldsymbol{p}<\mathbf{0 . 0 0 1}\end{array}$ & $\begin{array}{c}\mathrm{F}(1.5,212) \\
0.12 \\
\mathbf{p}=\mathbf{0 . 8 3}\end{array}$ \\
\hline cAROM transversal & $84 ; 79$ & $\begin{array}{c}\mathrm{F}(1,161) \\
0.11 \\
\mathbf{p}=\mathbf{0 . 7 4}\end{array}$ & $\begin{array}{c}\mathrm{F}(1.8,296) \\
1.06 \\
\mathbf{p}=\mathbf{0 . 3 5}\end{array}$ & $\begin{array}{c}\mathrm{F}(1.8,296) \\
0.60 \\
\mathbf{p}=\mathbf{0 . 5 5}\end{array}$ & $60 ; 79$ & $\begin{array}{c}\mathrm{F}(1,137) \\
0.15 \\
\mathbf{p}=\mathbf{0 . 7 0}\end{array}$ & $\begin{array}{c}\mathrm{F}(1.8,248) \\
1.31 \\
\mathbf{p}=\mathbf{0 . 2 7}\end{array}$ & $\begin{array}{c}\mathrm{F}(1,248) \\
0.86 \\
\mathbf{p}=\mathbf{0 . 4 2}\end{array}$ \\
\hline cAROM frontal & $84 ; 79$ & $\begin{array}{c}\mathrm{F}(1,161) \\
1.05 \\
\mathbf{p}=\mathbf{0 . 3 1}\end{array}$ & $\begin{array}{c}\mathrm{F}(1.8,288) \\
11.52 \\
\boldsymbol{p}<\mathbf{0 . 0 0 1}\end{array}$ & $\begin{array}{c}\mathrm{F}(1.8,288) \\
0.09 \\
\mathbf{p}=\mathbf{0 . 9 0}\end{array}$ & $60 ; 79$ & $\begin{array}{c}\mathrm{F}(1,137) \\
0.44 \\
\mathbf{p}=\mathbf{0 . 5 1}\end{array}$ & $\begin{array}{c}\mathrm{F}(1.8,243) \\
11.87 \\
\boldsymbol{p}<\mathbf{0 . 0 0 1}\end{array}$ & $\begin{array}{c}\mathrm{F}(1.8,243) \\
0.33 \\
\mathbf{p}=\mathbf{0 . 6 9}\end{array}$ \\
\hline Log (SES) & $86 ; 80$ & $\begin{array}{c}F(1,164) \\
0.02 \\
\mathbf{p}=\mathbf{0 . 9 0}\end{array}$ & $\begin{array}{c}\mathrm{F}(1,164) \\
71.40 \\
\boldsymbol{p}<\mathbf{0 . 0 0 1}\end{array}$ & $\begin{array}{c}F(1,164) \\
0.82 \\
\mathbf{p}=\mathbf{0 . 7 8}\end{array}$ & $62 ; 80$ & $\begin{array}{c}\mathrm{F}(1,140) \\
2.36 \\
\mathbf{p}=\mathbf{0 . 1 3}\end{array}$ & $\begin{array}{c}\mathrm{F}(1,140) \\
73.96 \\
\boldsymbol{p}<\mathbf{0 . 0 0 1}\end{array}$ & $\begin{array}{c}\mathrm{F}(1,140) \\
0.02 \\
\mathbf{p}=\mathbf{0 . 8 8}\end{array}$ \\
\hline Log (CSQ_CAT) & $56 ; 42$ & $\begin{array}{c}\mathrm{F}(1,96) \\
2.50 \\
\mathbf{p}=\mathbf{0 . 1 2}\end{array}$ & $\begin{array}{c}\mathrm{F}(1.9,180) \\
17.40 \\
\boldsymbol{p}<\boldsymbol{0 . 0 0 1}\end{array}$ & $\begin{array}{c}\mathrm{F}(1.9,180) \\
1.41 \\
\mathbf{p}=\mathbf{0 . 2 5}\end{array}$ & $43 ; 42$ & $\begin{array}{c}\mathrm{F}(1,83) \\
3.90 \\
\boldsymbol{p}=\mathbf{0 . 0 5}\end{array}$ & $\begin{array}{c}\mathrm{F}(2,166) \\
17.20 \\
\boldsymbol{p}<\boldsymbol{0 . 0 0 1}\end{array}$ & $\begin{array}{c}\mathrm{F}(2,166) \\
1.86 \\
\mathbf{p}=\mathbf{0 . 1 6}\end{array}$ \\
\hline Log (CSQ_COP) & $76 ; 68$ & $\mathrm{~F}(1,142)$ & $\mathrm{F}(1.8,253)$ & $\mathrm{F}(1.8,253)$ & $58 ; 68$ & $\mathrm{~F}(1,124)$ & $\mathrm{F}(1.8,225)$ & $\mathrm{F}(1.8,225)$ \\
\hline
\end{tabular}




\begin{tabular}{|c|c|c|c|c|c|c|c|c|}
\hline & & $\begin{array}{c}0.66 \\
\mathbf{p}=\mathbf{0 . 4 2}\end{array}$ & $\begin{array}{c}10.40 \\
p<0.001\end{array}$ & $\begin{array}{c}0.82 \\
\mathbf{p}=\mathbf{0 . 4 3}\end{array}$ & & $\begin{array}{c}0.39 \\
\mathbf{p}=\mathbf{0 . 5 3}\end{array}$ & $\begin{array}{c}12.0 \\
\boldsymbol{p}<\mathbf{0 . 0 0 1}\end{array}$ & $\begin{array}{c}0.89 \\
\mathbf{p}=\mathbf{0 . 4 0}\end{array}$ \\
\hline Log (CSQ_ADP) & $66 ; 64$ & $\begin{array}{c}\mathrm{F}(1,128) \\
0.43 \\
\mathbf{p}=\mathbf{0 . 5 1}\end{array}$ & $\begin{array}{c}\mathrm{F}(1.8,226) \\
27.10 \\
\boldsymbol{p}<\mathbf{0 . 0 0 1}\end{array}$ & $\begin{array}{c}\mathrm{F}(1.8,226) \\
0.32 \\
\mathbf{p}=\mathbf{0 . 7 3}\end{array}$ & $52 ; 64$ & $\begin{array}{c}\mathrm{F}(1,114) \\
1.0 \\
\mathbf{p}=\mathbf{0 . 3 1}\end{array}$ & $\begin{array}{c}\mathrm{F}(1.8,204) \\
22.8 \\
\boldsymbol{p}<\mathbf{0 . 0 0 1}\end{array}$ & $\begin{array}{c}\mathrm{F}(1.8,204) \\
0.51 \\
\mathbf{p}=\mathbf{0 . 5 8}\end{array}$ \\
\hline
\end{tabular}

${ }^{1}$ SPT: structured postoperative physiotherapy; ${ }^{2}$ SPT $>50 \%$ : patients with at least $50 \%$ attendance to treatment sessions in the structured postoperative physiotherapy group; ${ }^{3} \mathrm{SA}$ : standard postoperative approach

NME: neck muscle endurance in flexion and extension in seconds; cAROM: active cervical range of motion; SES: self-efficacy scale; CSQ: coping strategy questionnaire; CAT: catastrophizing subscale of the CSQ; COP: control over pain subscale of the CSQ; ADP: ability to decrease pain subscale of the CSQ. The results from repeated measures analysis of variance (ANOVA) are presented with F-values (degrees of freedom, error) and p-values. Non-normally distributed interval data and ordinal data were log-transformed prior to ANOVA. 

inclusion criteria and included after informed consent $(n=202)$

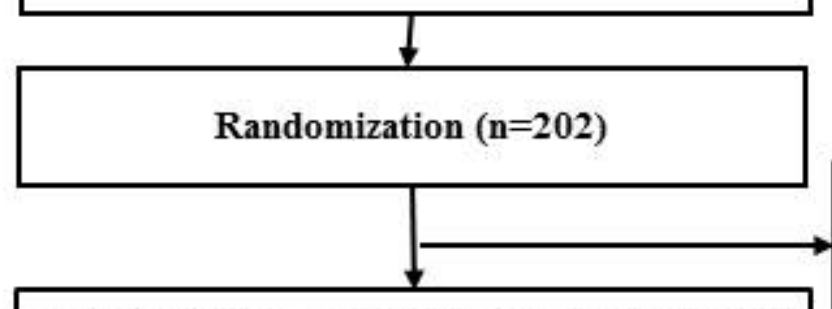

Excluded due to cancelled surgery $(n=1)$

\section{Structured postoperative physiotherapy}

- Allocated to intervention $(n=101)$

- Did not receive allocated intervention $(n=29)$; reasons: never started $(n=10)$, interrupted $(n=19)$

- Missing reports on attendance $(n=5)$

- $\geq 50 \%$ attendance to treatment session $(\mathrm{SPT} \geq 50 \% \mathrm{n}=67)$

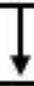

Follow-up at 3-month

Neck-related physical function ( $\mathrm{n}=95)$, Lost to follow-up unknown reasons $(n=6)$;

Self-efficacy and coping strategies ( $\mathrm{n}=85$ -

87), Lost to follow-up unknown reasons ( $n=$ 14-16)

Follow-up at 6-month

Neck-related physical function $(n=84-89)$, Lost to follow-up unknown reasons $(n=12$ 17);

Self-efficacy and coping strategies $(\mathrm{n}=88$ 90), Lost to follow-up unknown reasons( $n=$ 11-13)

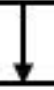

Neck-related physical function $(\mathrm{n}=83-84)$, $\mathrm{SPT} \geq 50 \%(\mathrm{n}=58-60)$

Self-efficacy and coping strategies $(n=56-$ 86), $\mathrm{SPT} \geq 50 \%(\mathrm{n}=43-62)$
Standard postoperative approach

- Allocated to intervention $(\mathrm{n}=100)$

\section{Follow-up at 3-month}

Neck-related physical function ( $\mathrm{n}=93)$, Lost to follow-up unlonown reasons $(n=7)$;

Self-efficacy and coping strategies $(\mathrm{n}=78$ -

80), Lost to follow-up unknown reasons ( $n=$ 20-22)

Follow-up at 6-month

Neck-related physical function ( $\mathrm{n}=81-82)$,

Lost to follow-up unknown reasons ( $n=18$ 19);

Self-efficacy and coping strategies $(\mathrm{n}=79$ -

81), Lost to follow-up unknown reasons ( $n=$ 19-21)

Neck-related physical function $(n=76-79)$

Self-efficacy and coping strategies $(n=42$ 80) 
- Non-resistance exercises to activate the deep neck

13 weeks

14. 16 weeks

17-18 weeks muscles (2-3 times daily)

- Isometric exercises to improve neuromuscular control and endurance of the deep neck muscles (2-3 times daily)

- Graded low-load endurance exercises for the neck, trunk and scapula muscles to improve neuromuscular control and endurance ( 1 time daily)

19-20 weeks

21-22 weeks

23-27 weeks

28-32 weeks

- Patients were encouraged to perform home exercises between sessions and after discharge, as well as to increase their overall activity level
- Relaxation, body awareness exercises

(+ vestibular rehabilitation)

postoperative

approach $=$

advise to contact primary healthcare centers for additional pragmatic treatments

when needed

- Activity goal setting and patient education on healing and pain processes

- Continued patient education on

- Breathing exercises pain processes

- Dialogue about personal activity level and pacing

- Dialogue about coping strategies for pain and stress management

- Dialogue the importance of social support in stress management

- Patient education and implications regarding ergonomy and physical activity

- Reinforcement of pain and stress management strategies

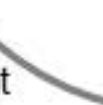

洒 